\title{
Constraint Solving Order in Position Based Dynamics
}

\author{
Yufeng Gu*, Kejian Yang and Cong Lu \\ Computer Science and Technology, Wuhan University of Technology, Wuhan, Hubei 430063, China \\ *Corresponding author
}

\begin{abstract}
In order to solve the problem that the adjustment sequence depends only on the data structure in the process of constraint satisfaction, a method of propagating from the fixed points is proposed. The method analyzes the internal force of fabrics in the real world, sorts the particles in ascending order according to the distance from the mass to the fixed points by using the breadth-first search method. The experimental results show that applying the proposed method to the position based dynamics simulation of cloth can converge faster and improve the simulation efficiency.
\end{abstract} order

Keywords- position based dynamics; cloth modelling; constraint

\section{INTRODUCTION}

Cloth simulation, which is widely used in digital entertainment, 3D games, garment industry and other fields, is a popular research direction of computer graphics. The purpose of cloth simulation is to build cloth model to generate realistic and real-time cloth animation.

In the early days of cloth modeling, efficient geometric method were widely used due to hardware limitations [1, 2]. With the development of computer hardware and graphics, the physical method which has a better reality based on Newton's law of motion becomes the mainstream. Physical method has developed a continuum model [3,4] and discrete particle model[5]. Because the discrete particle model uses the relatively simple mechanical form to describe the dynamic characteristics of cloth deformation, the computational complexity is very low, which makes the method performs better in real-time than the continuum model. The discrete particle model includes the mass-spring model [6], position based dynamics $[7,8]$, adaptive mesh method $[9,10]$ and other methods.

\section{POSITION BASED DYNAMICS}

In this paper, the position based dynamics method, which is fast, unconditionally stable and controllable, is used to simulate cloth. Although the simulation results are not as accurate as the force-based methods, the position based dynamics can guarantee a reasonable visual effect very quickly. This method is highly applicable for applications where require very high simulation speeds like virtual-reality, computer games, and movie effects. This method directly controls the displacements of particles by means of constraint projection. These constraints are defined by geometric criteria, which are simple and general and satisfy the conservation of angular and linear momentum.

\section{A. Algorithm Description}

T The cloth model can be described as a set of $N$ particles and $M$ constraints. A particle $i \in[1, \ldots, N]$ has a mass $m_{i}$, a position $\boldsymbol{x}_{i}$ and a velocity $\boldsymbol{v}_{i}, n_{j}$ is the cardinality. $C_{j}: R^{3 n_{j}} \rightarrow R$ represents the constraint equation. Based on this data and a time step $\Delta t$, the dynamic cloth is simulated as follows:

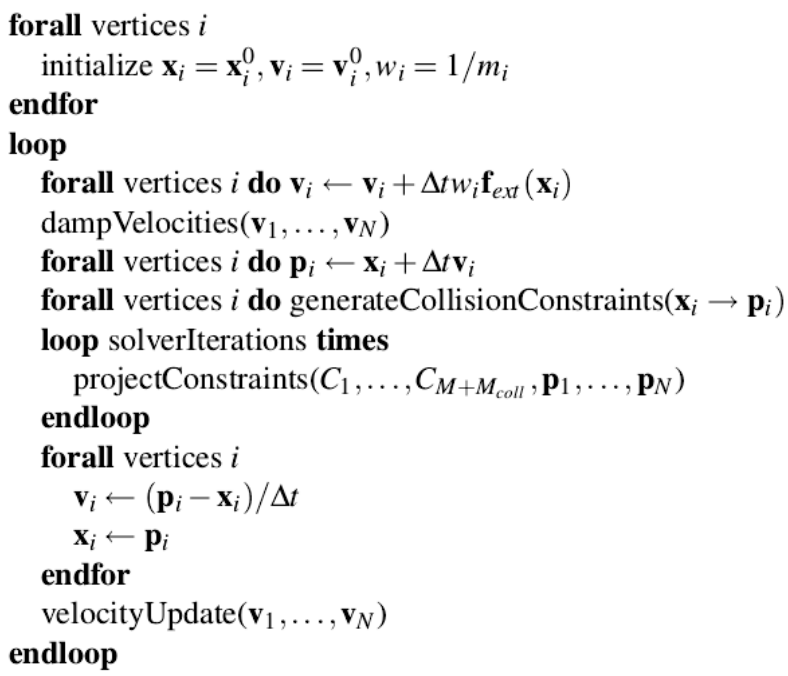

The particle mass, velocity and position are first initialized, and then the particle position is calculated by explicit Euler integration. This position is inaccurate and needs to be adjusted by an iterative step to update the position of each particle to satisfy the constraint condition and get the most accurate position. The velocity of each particle is due to the external force, for example, the velocity of a particle will change when the object is subjected to gravity, and if necessary, can also join the role of wind and friction.

\section{B. Constraint Projection}

After a numerical solution, all the particle positions $\boldsymbol{P}_{1}, \ldots, \boldsymbol{P}_{N}$ and $\boldsymbol{P}+\boldsymbol{P}_{\text {coll }}$ constraints are obtained. The constraint projection is used to determine the latest velocity and position of each cloth particle. The form of constraint is determined by stretching, bending or collision. Each constraint has its own specific constraint equation, and constraint projection means moving the points such that they satisfy the constraint. The most important issue in this step is the conservation of linear and angular momentum. Linear and angular momentum is conserved if

$$
\sum_{i} m_{i} \Delta \boldsymbol{P}_{i}=0
$$




$$
\sum_{i} \boldsymbol{r}_{i} m_{i} \Delta \boldsymbol{P}_{i}=0
$$

where $\Delta \boldsymbol{P}_{i}$ is the displacement of particle $i$ and $\boldsymbol{r}_{i}$ is the distance of the $\boldsymbol{P}_{i}$ to an arbitrary common rotation center. As shown in Table 1, stretching, bending, and collision constraints are typically used in the simulation. Common constraint types.

TABLE I. COMMON CONSTRAINT TYPES

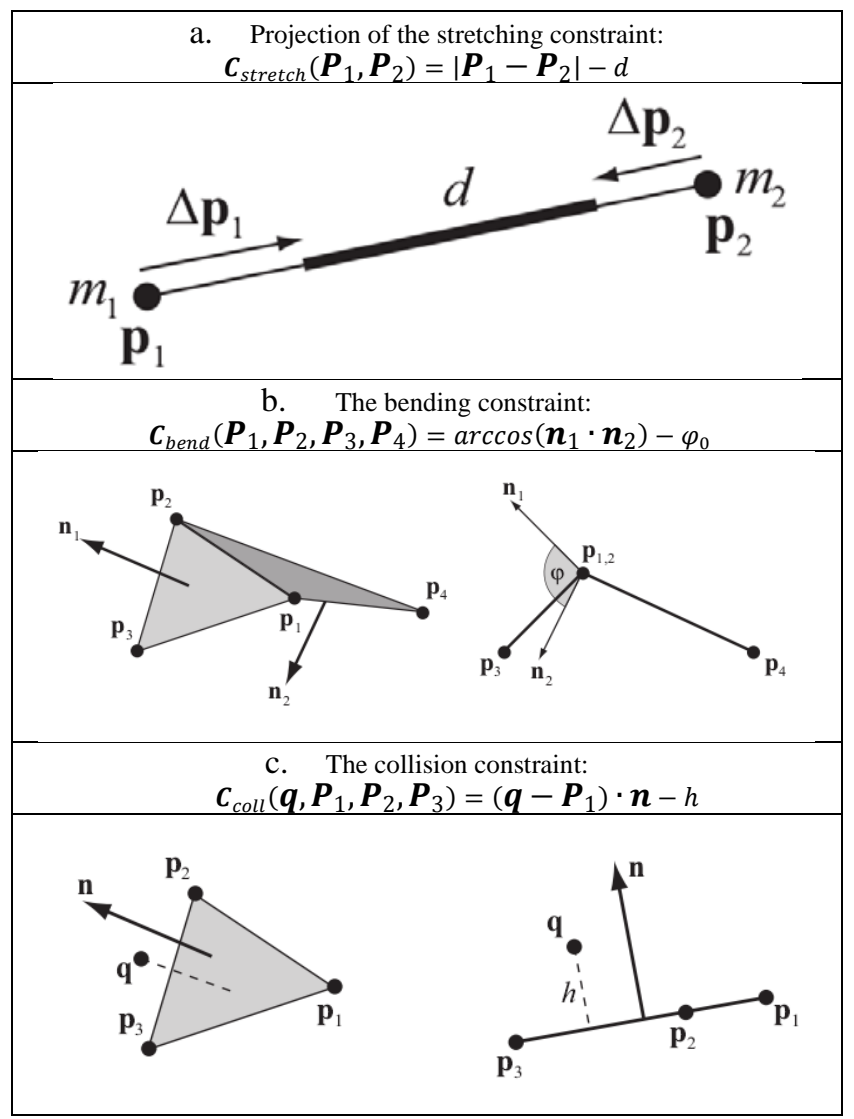

III. THE CONSTRAINT ORDER

The position based dynamics points out that the effect of constraint solving depends on the order in which constraints are solved. In over-constrained situations, the process can lead to oscillations if the order is not kept constant. However, there is no reasonable order given in this paper. Usually, their processing order depends on the data structure.

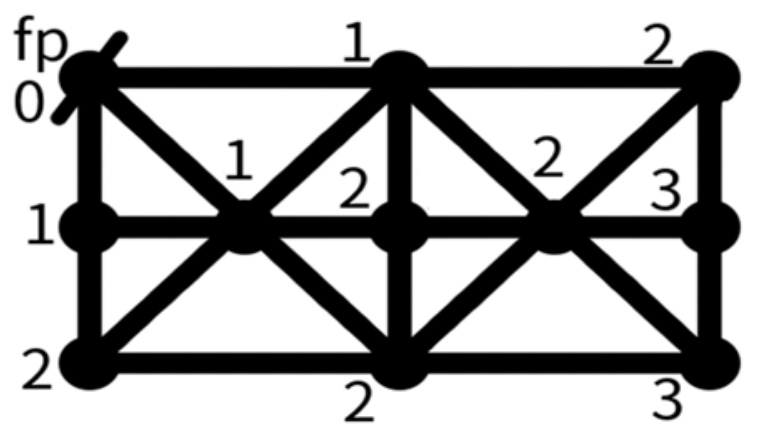

FIGURE I. THE DISTANCE FROM THE PARTICLE TO THE FIXED POINT

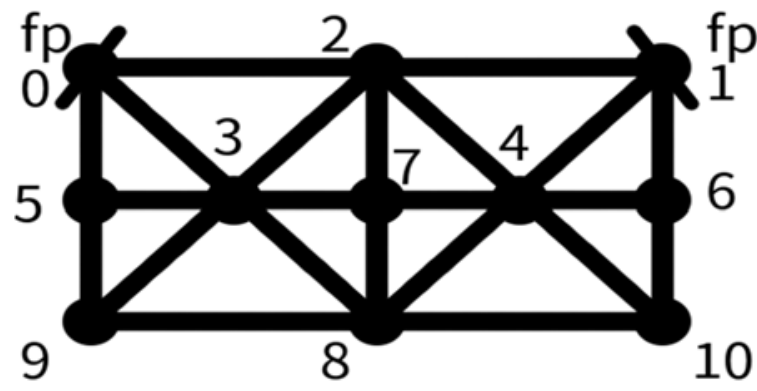

FIGURE II. PARTICLE PROCESSING SEQUENCE WITH TWO FIXED POINTS

Out purpose is to propose an appropriate constraint adjustment sequence to minimize the dissatisfaction of already corrected constraints because of another constraint adjustment, and so that the system can reach a state of equilibrium in a shorter time. The ordering is mainly aimed at the stretching constraint, because bending and collision are unpredictable.

Fixed points are very important in the process. Fixed points are points fixed on the object, but more generally, the fixed point is not always stationary, like the points fixed on the moving flagpole.

The process has two main stages. The first step determines the order in which the particles are processed. The second step sorts the constraints according to the information obtained in the first step.

When sorting particles, we use the idea of breadth-first search, recording the distance of each particle to each fixed point. The search is carried out from the fixed point, each fixed point to its own distance is 0 . According to the specific case of triangulation, the distance of the point that directly connected to the fixed one is 1 , and search each depth, plus one distance. As shown in Figure 1, "fp" is a fixed point, the numbers marked out are distances to the fixed point.

For each point, we have the distance to each fixed point, so the minimal distance to the nearest fixed point and a cumulative distance parameter can be obtained. All particles are then sorted according to the minimal distance. And for the particles with the same minimal distance, they are arranged in ascending order according to their cumulative distance parameters, which means the points "closest" to all fixed points processed first. Figure 2 shows the sequence of particle processing with two fixed points applying the stage one.

The second stage establishes the constraint ordered list based on the ordered particles of the first stage. From the beginning of the sorted particles, if the particle to which it is attached precedes it, the constraint on these two points is added to the constraint ordered list, otherwise do not add. For cases where more than one constraint needs to be added for a particle, the constraint associated with the preceding particle is preferentially added.

This idea is to simulate the actual behavior of cloth in reality that no over-elongation after deformation occurs and the process of stretching develops much like propagation from the points which contact with other objects. 


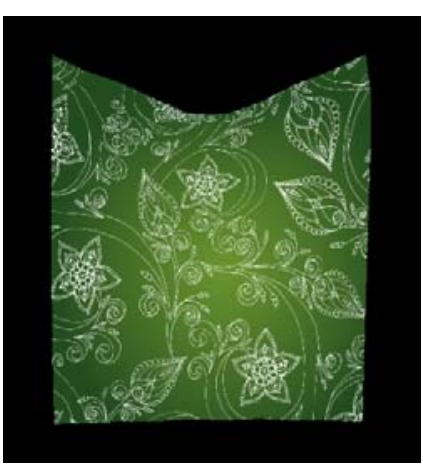

$\mathrm{a}$

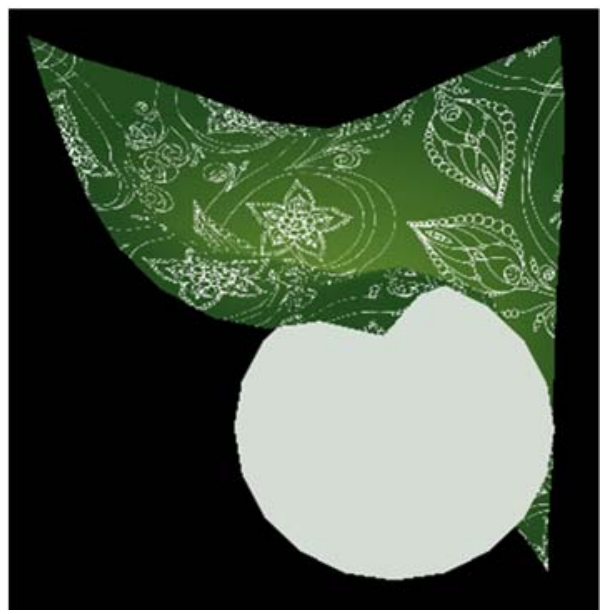

C

FIGURE III. FABRIC SIMULATION USING THE PROPOSED TECHNIQUE

\section{EXPERIMENTAL RESULTS}

Applying the proposed method to the cloth simulation system, the experimental results obtained shown in Figure 3, the figure a, b, c respectively show the hanging cloth, cloth in the wind and the collision with sphere. It shows that the cloth animation as a whole works well and there is no oscillation or distortion.

It was found that different triangulations had a slight effect on the final state of the fabric. Because in the process of constraint satisfaction, according to the different order of constraint adjustment, the displacements of some particles pulled by constraint will accumulate morphological changes. A very simple triangulation of the cloth model is used to amplify this phenomenon. In the wireframe of the cloth model, parts of the adjustment order of the constraints are marked out. Figure 4 shows the simulation results using the two techniques and their wireframes. And in the frames, parts of the constraint adjustment order are marked out. The four previous figures does not use the proposed method, the constraint adjustment order only depends on the data structure. In this implementation, the order is from left to right, from bottom to top(parts of the order marked out in the wireframe). The final drape shape of the fabric has a pulling effect which makes the simulation looks unreal. When using the proposed arrangement method shown in the next four figures, for the same triangulation, the final drape shape looks more realistic.
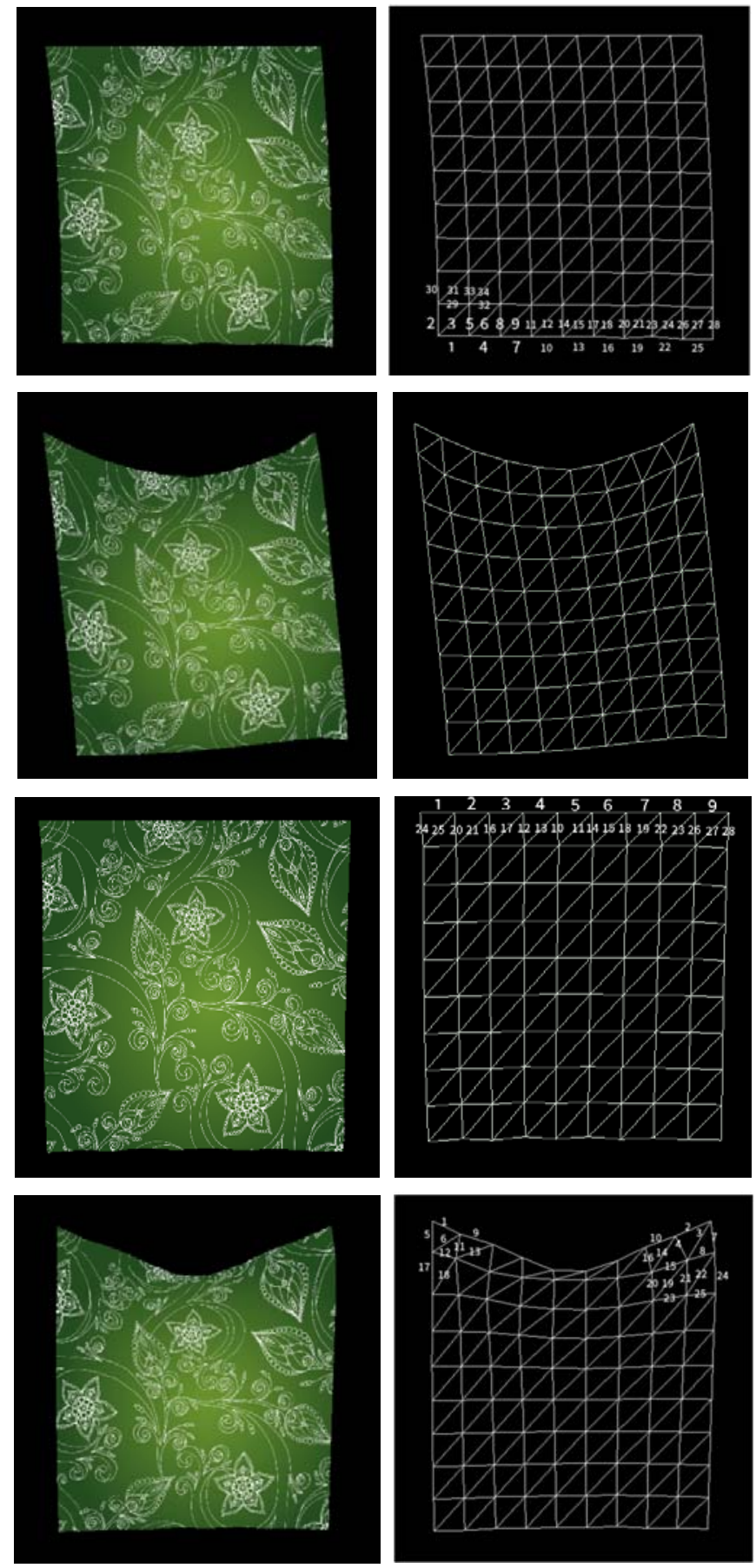

FIGURE IV. THE TEST RESULTS OF TWO KINDS OF TECHNOLOGY AND THEIR WIREFRAMES

Table 2 shows the time taken for the cloth to reach its equilibrium state from the initial state with and without the proposed method. The results show that the computational effectiveness of the new technique is better than the original method. Although there are complex and demanding 
computational procedures in obtaining an ordered list of constraints, this is only done once during program initialization. Once the ordered list is obtained, it can be used directly in next procedures. There is no need to repeat the sorting process of the constraints unless the fixed points change which does not occur frequently. And when constraints solved from the fixed points, particles can be projected to a position closer to the correct position, which reduces the number of iterations and makes the system converge more quickly.

TABLE II. PERFORMANCE DATA

\begin{tabular}{|c|c|c|c|c|c|}
\hline $\begin{array}{c}\text { Number of } \\
\text { Particles }\end{array}$ & $10 \times 10$ & $20 \times 20$ & $30 \times 30$ & $40 \times 40$ & $50 \times 50$ \\
\hline $\begin{array}{c}\text { Number of } \\
\text { Constraints }\end{array}$ & 261 & 1121 & 2581 & 4641 & 7301 \\
\hline $\begin{array}{c}\text { The original } \\
\text { method(ms) }\end{array}$ & 62 & 239 & 524 & 982 & 1573 \\
\hline $\begin{array}{c}\text { The proposed } \\
\text { method(ms) }\end{array}$ & 51 & 198 & 451 & 856 & 1362 \\
\hline
\end{tabular}

\section{CONCLUSIONS}

Realistic and efficient simulation of cloth can enhance the experience of the virtual world built by computers. Realism and real-time are always the key problem in cloth simulation. Based on the PBD method, we analyze the internal force of cloth, proposed a method sorting the constraints which begins form the fixed points propagating to all other particles. It is proved that the method is suitable for the PBD method, and has better realism and simulation efficiency.

\section{REFERENCES}

[1] Lu, J., \& Zheng, C. (2014). Dynamic cloth simulation by isogeometric analysis. Computer Methods in Applied Mechanics \& Engineering, 268(1), 475-493.

[2] Stumpp, T., Spillmann, J., Becker, M., \& Teschner, M. (2008). A Geometric Deformation Model for Stable Cloth Simulation. The Workshop on Virtual Reality Interactions and Physical Simulations, Vriphys 2008, Grenoble, France (pp.39-46). DBLP.

[3] Volino P, Magnenat-Thalmann N, Faure F. A simple approach to nonlinear tensile stiffness for accurate cloth simulation[J]. Acm Transactions on Graphics, 2009, 28(4):1-16.

[4] Carignan, M., Yang, Y., Thalmann, N. M., \& Thalmann, D. (1992). Dressing animated synthetic actors with complex deformable clothes. Acm Siggraph Computer Graphics, 26(2), 99-104.

[5] Breen, D. E. (1999). Predicting the drape of woven cloth using interacting particles. Proceedings of the 21st annual conference on Computer graphics and interactive techniques (Vol.39, pp.365-372). ACM.

[6] Provot, X. (2001). Deformation constraints in a mass-spring model to describe rigid cloth behavior.

[7] Müller, M., Heidelberger, B., Hennix, M., \& Ratcliff, J. (2007). Position based dynamics $\hbar$. Journal of Visual Communication \& Image Representation, 18(2), 109-118.

[8] Francu, M., \& Moldoveanu, F. (2015). Cloth Simulation Using Soft Constraints. Wscg.

[9] Li, L., \& Volkov, V. (2005). Cloth animation with adaptively refined meshes. Twenty-Eighth Australasian Conference on Computer Science (pp.107-113). Australian Computer Society, Inc.

[10] Oliveira, S. M. F. D. (2014). Cloth Simulation with Triangular Mesh Adaptivity. Sibgrapi Conference on Graphics (pp.235-242). 